\title{
Eficiência de diferentes bicos e volumes de calda no controle de tripes em cebola.
}

\author{
Paulo Antônio de S. Gonçalves ${ }^{1}$; Luiz A. Palladini ${ }^{2}$ \\ EPAGRI - Estação Experimental de Ituporanga, C. Postal 121, 88.400-000, Ituporanga - SC; \\ ${ }_{2}$ EPAGRI - Estação Experimental de Caçador, C. Postal 591, 8.9500-000, Caçador - SC. e-mail: eeitu@epagri.rct-sc.br
}

\section{RESUMO}

O objetivo do trabalho foi avaliar a eficiência de diferentes volumes de calda e tipo de bico no controle químico de Thrips tabaci em cebola. Dois experimentos foram conduzidos na EPAGRI, Estação Experimental de Ituporanga, SC, no período de agosto a dezembro de 1996 e 1997. Os tratamentos com bico leque e respectivos níveis de vazão foram XR $110015 \mathrm{VS}^{\circledR}$ - $236 \mathrm{~L} / \mathrm{ha}$, XR $11002 \mathrm{VS}^{\circledR}$ - $316 \mathrm{~L} / \mathrm{ha}$, XR $11003 \mathrm{VS}^{\circledR}$ - $472 \mathrm{~L} / \mathrm{ha}$, XR $11004 \mathrm{VS}^{\circledR}$ - $632 \mathrm{~L} / \mathrm{ha}$, XR $11005 \mathrm{VS}^{\circledR}-788$ L/ha, TJ $6011002 \mathrm{VS}^{\circledR}$ - $316 \mathrm{~L} / \mathrm{ha}$, TJ 60110 $04 \mathrm{VS}^{\circledR}$ - $632 \mathrm{~L} / \mathrm{ha}$; com bico cone foram Conejet TSVS ${ }^{\circledR}-236 \mathrm{~L} / \mathrm{ha}$, Conejet TXVK $18^{\circledR}$ - $472 \mathrm{~L} / \mathrm{ha}$, Conejet TXVK $26^{\circledR}$ - $632 \mathrm{~L} / \mathrm{ha}$, D6 Difusor V5 ${ }^{\circledR}-600 \mathrm{~L} /$ ha, além da testemunha, sem tratamento. O delineamento experimental utilizado foi blocos ao acaso com quatro repetições. O tamanho de parcela foi de $2,8 \mathrm{~m} \mathrm{x} \mathrm{3,0} \mathrm{m.} \mathrm{O} \mathrm{inseti-}$ cida usado foi clorpirifós 0,72 g. i.a./ha. A amostragem de ninfas de T. tabaci foi realizada no campo em cinco plantas escolhidas ao acaso em cada parcela. A redução populacional de tripes foi semelhante entre os diferentes volumes de calda e tipos de bico utilizados. Portanto, os bicos cone e leque aplicando volumes de calda entre 236 a $788 \mathrm{~L} / \mathrm{ha}$, apresentaram a mesma eficiência no controle de $T$. tabaci em cebola.

Palavras- chave: Allium cepa, tripes, controle químico, bicos, volumes.

\section{ABSTRACT}

Efficiency of different nozzle types and volume of the insecticide solution in the control of thrips in onions.

The objective of this work was to evaluate the efficiency of different nozzle types and volume of the insecticide solution in controlling thrips (Thrips tabaci) in onions. The work was carried out from August to December, 1996 and 1997. The treatments consisted of different nozzle types (fan and cone) and different flow rates. Fan nozzles were XR $110015 \mathrm{VS}^{\circledR}$ - $236 \mathrm{~L} / \mathrm{ha}$, XR 11002 $\mathrm{VS}^{\circledR}$ - $316 \mathrm{~L} / \mathrm{ha}, \mathrm{XR} 11003 \mathrm{VS}^{\circledR}$ - $472 \mathrm{~L} / \mathrm{ha}, \mathrm{XR} 11004 \mathrm{VS}^{\circledR}-632 \mathrm{~L} /$ ha, XR $11005 \mathrm{VS}^{\circledR}$ - $788 \mathrm{~L} / \mathrm{ha}$, TJ $6011002 \mathrm{VS}^{\circledR}-316 \mathrm{~L} / \mathrm{ha}$, TJ 60 11004 VS - $632 \mathrm{~L} / \mathrm{ha}$; and cone nozzles were Conejet TSVS ${ }^{\circledR}-236$ L/ha, Conejet TXVK $18^{\circledR}-472 \mathrm{~L} / \mathrm{ha}$, Conejet TXVK $26^{\circledR}-632 \mathrm{~L} /$ ha, D6 Difusor V5 ${ }^{\circledR}-600 \mathrm{~L} / \mathrm{ha}$. Besides these treatments there was an untreated check. The experimental design was of randomized complete blocks with four replications. The insecticide used was chlorpyrifos 0.72 g.a.i./ha. The evaluation of the number of nimphs of $T$. tabaci was made weekly in the field using five plants per plot. The fan or cone nozzle applying $236 \mathrm{~L} / \mathrm{ha}$ to $788 \mathrm{~L} / \mathrm{ha}$ of the insecticide solution (chlorpyrifos) gave similar thrips control.

Keywords: Allium cepa, thrips, chemical control, nozzles, volumes.

(Aceito para publicação em 16 de maio de 2.000)

$\mathrm{O}$ tripes, Thrips tabaci Lindeman, é a principal praga da cultura da cebola no Estado de Santa Catarina. Este inseto alimenta-se principalmente das folhas, causando danos na região da bainha. Para o seu controle os produtores estão utilizando tecnologia de aplicação de agrotóxicos bastante diversificada principalmente quanto ao volume de calda de inseticidas, variando-se de aproximadamente $200 \mathrm{~L} /$ ha com pulverizador costal a $800 \mathrm{~L} / \mathrm{ha}$ com pulverizadores tratorizados, utilizando tanto bicos tipo leque quanto cone. Nos trabalhos experimentais de controle químico de tripes em cebola, os volumes de calda também apresentam variações: Lorca (1958) utilizou $760 \mathrm{~L} / \mathrm{ha}$; Sharma \& Srivastava (1965) 371 a $494 \mathrm{~L} / \mathrm{ha}$; Howland \& Wilcox (1966) 110 L/ha, com pulverizador de alta pressão e 550 L/ha, com pulverizador manual; Raheja (1973) 110 L/ha; Mote (1976) 500, 600 e $800 \mathrm{~L} / \mathrm{ha}$; Rossiter \& Geisemann (1976) utilizaram no primeiro ano 1685 $\mathrm{L} / \mathrm{ha}$, reduzindo para $1120 \mathrm{~L} / \mathrm{ha}$ no ano seguinte; Mayer et al . (1987) $151 \mathrm{~L} / \mathrm{ha}$; Saini et al. (1989) 746 L/ha. Segundo Rossiter (1980) citado por Sato (1989) os inseticidas devem ser aplicados com uma quantidade de água suficiente para molhar toda a planta, preferencialmente, esta quantidade não deve ser inferior a $600 \mathrm{~L} / \mathrm{ha}$, para atingir as ninfas que ficam alojadas na parte interna das folhas. Em seus trabalhos, Sato (1989) utilizou o volume de $500 \mathrm{~L} / \mathrm{ha}$. Gonçalves \& Guimarães (1995), para tentar atingir os insetos alojados na parte interna das folhas, utilizaram aproximadamente $600 \mathrm{~L} / \mathrm{ha}$, recomendando bicos leque 80.03 ou 110.04 , pois apresentaram controle eficaz em experimentos realizados na EPAGRI, Estação Experimental de Ituporanga, SC. Em relação ao tipo de bico, Guedes et al . (1982) e
Cavalcante et al. (1986) também utilizaram bicos leque e obtiveram redução populacional significativa de $T$. tabaci.

O objetivo deste trabalho foi determinar o volume de calda e o tipo de bico adequado para o controle químico de tripes na cultura da cebola, para as condições do Alto Vale do Itajaí, SC.

\section{MATERIAL E MÉTODOS}

Dois experimentos foram conduzidos na Estação Experimental de Ituporanga, EPAGRI (latitude $27^{\circ}, 22^{\prime \prime}$ $\mathrm{S}$, longitude $49^{\circ}, 35^{\prime} \mathrm{W}$ ), no período de agosto a dezembro de 1996 e 1997 . Os transplantes foram realizados em 12/08 (1996) e 26/08 (1997), e a colheita em 04/12 (1996) e 15/12 (1997). Utilizouse mudas de cebola da cultivar Crioula, em parcelas de $2,8 \mathrm{~m} \times 3,0 \mathrm{~m}$, com espaçamento de $40 \mathrm{~cm} \times 7,5 \mathrm{~cm}$, totalizando 280 plantas por parcela. A 
Tabela 1. Número médio de ninfas de Thrips tabaci Lind. por planta $(\mathrm{N})$ e percentagem de eficiência (\%EF*), em cebola cultivar Crioula, para diferentes bicos e volumes de calda de inseticidas. Ituporanga, EPAGRI, 1996.

\begin{tabular}{|c|c|c|c|c|c|c|c|c|c|c|c|c|c|}
\hline \multirow{3}{*}{ Tratamentos } & \multicolumn{13}{|c|}{ Datas de avaliação } \\
\hline & \multirow{2}{*}{$\begin{array}{c}\begin{array}{c}14 / 10 \\
\text { (prévia) }\end{array} \\
\mathrm{N}\end{array}$} & \multicolumn{2}{|c|}{$17 / 10$} & \multicolumn{2}{|c|}{$25 / 10$} & \multicolumn{2}{|c|}{$31 / 10$} & \multicolumn{2}{|c|}{$07 / 11$} & \multicolumn{2}{|c|}{$13 / 11$} & \multicolumn{2}{|c|}{$21 / 11$} \\
\hline & & $\mathbf{N}$ & $\% \mathrm{EF}^{*}$ & $\mathbf{N}$ & $\% E F^{*}$ & $\mathbf{N}$ & $\% E F^{*}$ & $\mathbf{N}$ & $\% \mathrm{EF}^{*}$ & $\mathbf{N}$ & $\% \mathrm{EF}^{*}$ & $\mathbf{N}$ & \%EF* \\
\hline XR.110.015 VS - 236 L/ha & 15,4 a & $12,7 \mathrm{a}$ & 34,5 & $11,0 a b$ & 52,6 & $6,9 a b$ & 69,7 & $16,3 a b$ & 52,1 & $25,5 a b$ & 49,7 & $8,5 a b$ & 76,7 \\
\hline XR.110.02 VS - 316 L/ha & $14,6 a$ & $10,7 a$ & 44,9 & $6,4 \mathrm{~b}$ & 72,4 & $3,4 \quad b$ & 85,1 & $13,4 \quad b$ & 60,6 & $28,5 a b$ & 43,8 & $9,2 a b$ & 74,8 \\
\hline XR.110.03 VS - 472 L/ha & $14,1 \mathrm{a}$ & $10,2 \mathrm{a}$ & 47,4 & $9,5 a b$ & 59,1 & $4,1 \quad b$ & 82,0 & $7,6 \mathrm{~b}$ & 77,7 & $20,3 \quad b$ & 60,0 & $8,3 a b$ & 77,3 \\
\hline XR.110.04 VS - 632 L/ha & 20,0 a & $9,2 \mathrm{a}$ & 52,6 & $9,0 a b$ & 61,2 & $4,1 \quad b$ & 82,0 & $17,8 a b$ & 47,7 & $23,7 a b$ & 53,3 & $4,2 \mathrm{~b}$ & 88,5 \\
\hline XR.110.05 VS - 788 L/ha & $18,3 \mathrm{a}$ & $14,5 \mathrm{a}$ & 25,3 & $8,9 a b$ & 61,6 & $4,9 \quad b$ & 78,5 & $7,3 \mathrm{~b}$ & 78,5 & $21,6 a b$ & 57,4 & $6,6 a b$ & 81,9 \\
\hline TJ60 110.02 VS - 316 L/ha & $16,5 \mathrm{a}$ & 9,4 a & 51,6 & $6,1 \mathrm{~b}$ & 73,7 & $7,7 a b$ & 66,2 & $16,9 a b$ & 50,3 & $18,0 \quad b$ & 64,5 & $5,5 \mathrm{~b}$ & 84,9 \\
\hline TJ60 110.04 VS - 632 L/ha & $16,0 \mathrm{a}$ & $8,6 \mathrm{a}$ & 55,7 & $8,9 a b$ & 61,6 & $6,1 a b$ & 73,3 & $14,7 a b$ & 56,8 & $29,2 a b$ & 42,4 & $7,8 a b$ & 78,6 \\
\hline CONEJET TXVS 8 - 236 L/ha & 7,4 a & $9,2 \mathrm{a}$ & 52,6 & $11,9 a b$ & 48,7 & $7,1 a b$ & 68,9 & $15,1 a b$ & 55,6 & $34,1 a b$ & 32,7 & $12,2 a b$ & 66,6 \\
\hline CONEJET TXVK 18 - 472 L/ha & $12,3 \mathrm{a}$ & $9,2 \mathrm{a}$ & 52,6 & $16,0 \mathrm{ab}$ & 31,0 & $7,0 a b$ & 69,3 & $16,9 a b$ & 50,3 & $26,4 a b$ & 47,9 & $10,9 a b$ & 70,1 \\
\hline CONEJET TXVK 26 - 632 L/ha & 14,7 a & $12,6 \mathrm{a}$ & 35,1 & $11,9 a b$ & 48,7 & $4,6 \quad b$ & 79,8 & $13,6 b$ & 60,0 & $33,8 \mathrm{ab}$ & 33,3 & $7,5 a b$ & 79,5 \\
\hline D6 DIFUSOR V5 - 600 L/ha & $10,5 \mathrm{a}$ & $9,2 \mathrm{a}$ & 52,6 & $8,2 a b$ & 64,7 & $4,9 \quad b$ & 78,5 & $10,3 \mathrm{~b}$ & 69,7 & $23,4 a b$ & 53,9 & $5,4 \quad b$ & 85,2 \\
\hline Testemunha & $12,1 \mathrm{a}$ & $19,4 \mathrm{a}$ & - & $23,2 \mathrm{a}$ & - & $22,8 \mathrm{a}$ & - & $34,0 \mathrm{a}$ & - & $50,7 \mathrm{a}$ & - & $36,5 \mathrm{a}$ & - \\
\hline C.V. (\%) & 19,1 & 26,7 & & 20,7 & & 27,2 & & 13,9 & & 10,8 & & 32,7 & \\
\hline
\end{tabular}

OBS: Médias seguidas da mesma letra nas colunas não diferem entre si pelo teste de Tukey $(\mathrm{P}<0,05)$.

* Porcentagem de eficiência corrigida pela fórmula de Abbott (1925).

condução da cultura seguiu o proposto por EMPASC/ACARESC (1991). O delineamento experimental foi blocos ao acaso com 12 tratamentos e quatro repetições. As aplicações foram realizadas com pulverizador manual em pressão constante com $\mathrm{CO}_{2}$, a três bares. Os bicos e os volumes de calda utilizados foram: tipo leque, 1 - XR 110015 VSâ 236 L/ha; 2 - XR 11002 VSâ - 316 L/ ha; 3 - XR 11003 VSâ - 472 L/ha; 4 XR 11004 VSâ - 632 L/ha; 5 - XR 110 05 VSâ - 788 L/ha; 6 - TJ60 11002 VSâ - 316 L/ha; 7 - TJ60 11004 VSâ - 632 L/ha; tipo cone, 8 - Conejet TXVS - 8â - 236 L/ha; 9 - Conejet TXVK - 18â 472 L/ha; 10 - Conejet TXVK26â - 632 L/ha; 11 - D6 Difusor V5â - 600 L/ha; 12 - Testemunha sem tratamento. $\mathrm{O}$ inseticida usado foi clorpirifós na dosagem de 0,72 g.i.a./ha. Os tratamentos foram realizados nas seguintes datas $16 /$ $10,24 / 10,30 / 10,06 / 11,12 / 11$ e $20 / 11$ para o primeiro ensaio, em 1996 e $28 /$ $10,11 / 11,24 / 11$ e $03 / 12$ para o segundo ensaio, no ano de 1997.

O número de ninfas de tripes foi determinado no campo em todas as folhas, com lupa manual $75 \mathrm{~mm}$, aproximadamente 24 horas após as pulverizações. Para o cálculo da eficiência do inseticida utilizou-se a fórmula de Abbott (1925).

A produtividade foi avaliada em 60 bulbos por parcela, previamente determinados, selecionando-se dentre estes apenas aqueles com padrão comercial, ou seja com diâmetro acima de $4 \mathrm{~cm}$.

$\mathrm{Na}$ análise dos dados o número médio de ninfas de tripes foi transformado em $\log \mathrm{x}+0,5$ e a produtividade em $\mathrm{t} /$ ha. Os resultados foram submetidos a análise de variância e as médias comparadas pelo teste de Tukey a $5 \%$ de probabilidade.

\section{RESULTADOS E DISCUSSÃO}

As avaliações prévias do número de ninfas de tripes, para os anos de 1996 e 1997, não apresentaram diferenças estatísticas, indicando uniformidade na distribuição de ninfas em todas as parcelas experimentais. Após a primeira pulverização, verificou-se que a densidade populacional do inseto permaneceu semelhante em todos os tratamentos, nos dois ensaios (Tabelas 1 e 2), à exceção do tratamento XR.110.04 VS $632 \mathrm{~L} /$ ha que apresentou menor número de ninfas no segundo ensaio, quando comparado à testemunha (Tabela 2). Com as pulverizações subsequentes os bicos XR, TJ, CONEJET e D6, nos diferentes volumes, obtiveram níveis de controle não diferindo estatisticamente entre si, nos dois anos de avaliação (Tabelas 1 e 2). Portanto, os bicos leque e cone aplicando volumes de calda entre 236 a $788 \mathrm{~L} / \mathrm{ha}$, apresentaram a mesma eficiência para o controle químico de tripes em cebola. Estes resultados diferem da recomendação de Darcádia (1980) citado por Sato (1989) e Gonçalves e Guimarães (1995), que recomendaram volumes de calda acima de $600 \mathrm{~L} / \mathrm{ha}$, para o controle de tripes em cebola. Mayeux \& Wene (1950) utilizando inseticidas clorados (atualmente com seu uso proibido), concluíram que aplicações líquidas a baixo volume, tanto para equipamentos terrestre quanto aéreo foram tão eficientes quanto as formulações pó. De acordo com Carman (1975) no controle de ácaros e tripes deve-se considerar que estes não necessitam ser atingidos diretamente pelas gotas, pois durante os seus intensos movimentos eles entrarão em contanto com o produto, possibilitando assim, a redução de volumes de calda nos tratamentos.

A aplicação sucessiva de inseticidas no controle de tripes, não provocou reduções populacionais significativas em 
Tabela 2. Número médio de ninfas de Thrips tabaci Lind. por planta $(\mathrm{N})$ e percentagem de eficiência (\%EF*), em cebola cultivar Crioula, para diferentes bicos e volumes de calda de inseticidas. Ituporanga, EPAGRI, 1997.

\begin{tabular}{|c|c|c|c|c|c|c|c|c|c|}
\hline \multirow{3}{*}{ Tratamentos } & \multicolumn{9}{|c|}{ Datas de avaliação } \\
\hline & \multirow{2}{*}{$\begin{array}{c}\begin{array}{c}27 / 10 \\
\text { (prévia) }\end{array} \\
N\end{array}$} & \multicolumn{2}{|c|}{$31 / 10$} & \multicolumn{2}{|c|}{$12 / 11$} & \multicolumn{2}{|c|}{$25 / 11$} & \multicolumn{2}{|c|}{$04 / 12$} \\
\hline & & $\mathbf{N}$ & $\% E F *$ & $\mathbf{N}$ & $\% \mathrm{EF}^{*}$ & $\mathbf{N}$ & $\% \mathrm{EF}^{*}$ & $\mathbf{N}$ & $\% E F *$ \\
\hline XR.110.015 VS - 236 L/ha & $34,5 \mathrm{a}$ & $3,8 a b$ & 66,1 & $14,8 \quad b$ & 55,4 & $9,4 \quad b$ & 75,1 & $10,7 a b$ & 42,2 \\
\hline XR.110.02 VS - 316 L/ha & $27,8 \mathrm{a}$ & $4,7 a b$ & 58,0 & $15,9 \quad b$ & 52,1 & $10,4 \quad b$ & 72,5 & $8,6 a b$ & 53,5 \\
\hline XR.110.03 VS - 472 L/ha & $20,2 \mathrm{a}$ & $4,3 a b$ & 61,6 & $17,6 a b$ & 47,0 & $6,6 \mathrm{~b}$ & 82,5 & $3,3 \quad b$ & 82,2 \\
\hline XR.110.04 VS - 632 L/ha & $21,2 \mathrm{a}$ & $2,4 \quad b$ & 78,6 & $14,8 \quad b$ & 55,4 & $11,1 \quad b$ & 70,6 & $6,9 a b$ & 62,7 \\
\hline XR.110.05 VS - 788 L/ha & 29,3 a & $4,9 a b$ & 56,3 & $15,5 a b$ & 53,3 & $5,0 \quad b$ & 86,8 & $4,5 a b$ & 75,7 \\
\hline TJ60 110.02 VS - 316 L/ha & $31,6 \mathrm{a}$ & $6,0 a b$ & 46,4 & $16,0 \quad b$ & 51,8 & $14,9 a b$ & 60,6 & $6,0 a b$ & 67,6 \\
\hline TJ60 110.04 VS - 632 L/ha & 33,5 a & $7,0 a b$ & 37,5 & $16,2 a b$ & 51,2 & $5,0 \quad b$ & 86,8 & $4,0 \quad b$ & 78,4 \\
\hline CONEJET TXVS 8 - 236 L/ha & 22,7 a & $6,3 a b$ & 43,8 & $21,8 a b$ & 34,3 & $10,5 \mathrm{~b}$ & 72,2 & $8,9 a b$ & 51,9 \\
\hline CONEJET TXVK 18 - 472 L/ha & 23,9 a & $7,4 a b$ & 33,9 & $21,2 a b$ & 36,1 & $11,4 a b$ & 69,8 & $5,2 a b$ & 71,9 \\
\hline CONEJET TXVK2 6 - 632 L/ha & $26,2 \mathrm{a}$ & $6,7 a b$ & 40,2 & $15,3 a b$ & 53,9 & $8,5 \mathrm{~b}$ & 77,5 & $5,0 a b$ & 73,0 \\
\hline D6 DIFUSOR V5 - 600 L/ha & $19,7 \mathrm{a}$ & $5,7 a b$ & 49,1 & $21,6 a b$ & 34,9 & $8,4 \quad b$ & 77,8 & $5,3 a b$ & 71,4 \\
\hline Testemunha & $26,6 \mathrm{a}$ & $11,2 \mathrm{a}$ & - & $33,2 \mathrm{a}$ & - & $37,8 \mathrm{a}$ & - & $18,5 \mathrm{a}$ & - \\
\hline C.V. (\%) & 11,2 & 31,1 & & 10,6 & & 22,9 & & 32,2 & \\
\hline
\end{tabular}

OBS: Médias seguidas da mesma letra nas colunas não diferem entre si pelo teste de Tukey $(\mathrm{P}<0,05)$.

* Porcentagem de eficiência corrigida pela fórmula de Abbott (1925).

Tabela 3. Produtividade comercial média e peso comercial médio de bulbos de cebola, cultivar Crioula. Ituporanga, EPAGRI, 1996 e 1997.

\begin{tabular}{lrrcc}
\hline \multirow{2}{*}{\multicolumn{1}{c}{ Tratamentos }} & \multicolumn{2}{c}{$\begin{array}{c}\text { Produtividade } \\
\text { (t/ha) }\end{array}$} & \multicolumn{2}{c}{$\begin{array}{c}\text { Peso médio de } \\
\text { bulbos (g) }\end{array}$} \\
\cline { 2 - 6 } & $\mathbf{1 9 9 6}$ & $\mathbf{1 9 9 7}$ & $\mathbf{1 9 9 6}$ & $\mathbf{1 9 9 7}$ \\
\hline XR.110.015 VS - 236 L/ha & $9,3 \mathrm{a}$ & $16,9 \mathrm{a}$ & $58,7 \mathrm{a}$ & $61,1 \mathrm{ab}$ \\
XR.110.02 VS - 316 L/ha & $10,0 \mathrm{a}$ & $17,4 \mathrm{a}$ & $60,0 \mathrm{a}$ & $60,3 \mathrm{ab}$ \\
XR.110.03 VS - 472 L/ha & $9,9 \mathrm{a}$ & $16,0 \mathrm{a}$ & $63,6 \mathrm{a}$ & $60,1 \mathrm{ab}$ \\
XR.110.04 VS - 632 L/ha & $12,0 \mathrm{a}$ & $16,1 \mathrm{a}$ & $59,2 \mathrm{a}$ & $60,5 \mathrm{ab}$ \\
XR.110.05 VS - 788 L/ha & $9,0 \mathrm{a}$ & $18,8 \mathrm{a}$ & $58,5 \mathrm{a}$ & $63,7 \mathrm{a}$ \\
TJ60 110.02 VS - 316 L/ha & $10,9 \mathrm{a}$ & $14,6 \mathrm{a}$ & $60,2 \mathrm{a}$ & $57,0 \mathrm{~b}$ \\
TJ60 110.04 VS - 632 L/ha & $9,5 \mathrm{a}$ & $16,1 \mathrm{a}$ & $60,0 \mathrm{a}$ & $57,6 \mathrm{~b}$ \\
CONEJET TXVS 8 - 236 LI/ha & $11,8 \mathrm{a}$ & $17,3 \mathrm{a}$ & $62,3 \mathrm{a}$ & $62,2 \mathrm{ab}$ \\
CONEJET TXVK 18 - 472 L/ha & $11,7 \mathrm{a}$ & $16,3 \mathrm{a}$ & $61,9 \mathrm{a}$ & $59,5 \mathrm{ab}$ \\
CONEJET TXVK 26 - 632 L/ha & $11,0 \mathrm{a}$ & $17,7 \mathrm{a}$ & $60,1 \mathrm{a}$ & $61,9 \mathrm{ab}$ \\
D6 DIFUSOR V5 - 600 L/ha & $10,1 \mathrm{a}$ & $17,5 \mathrm{a}$ & $60,9 \mathrm{a}$ & $60,0 \mathrm{ab}$ \\
Testemunha & $7,9 \mathrm{a}$ & $17,1 \mathrm{a}$ & $56,4 \mathrm{a}$ & $59,8 \mathrm{ab}$ \\
\hline C.V. (\%) & 23,6 & 10,1 & 7,0 & 5,1 \\
\hline
\end{tabular}

OBS: Médias seguidas da mesma letra nas colunas não diferem entre si pelo teste de Tukey $(\mathrm{P}<0,05)$.

relação à testemunha (Tabelas 1 e 2). $\mathrm{O}$ controle químico foi dificultado provavelmente devido às condicões climáticas observadas nos meses de outubro e novembro em Ituporanga (SC), (altas temperaturas e baixas precipitações) serem favoráveis ao rápido desenvolvimento populacional do inseto (Gonçalves, 1997 b). Além disso, "pode" estar ocorrendo resistência do inseto, com aplicação exagerada de inseticidas pelos agricultores da região do Alto Vale do Itajaí, SC, exercendo uma pressão de seleção sobre a praga. Lewis, 1973, observou que T. tabaci apresentou resistência aos clorados no passado, portanto não deve ser descartada a possibilidade que o mesmo esteja ocorrendo com piretróides e fosforados.

Os tratamentos com volume reduzido de calda proporcionam diversas vantagens para o produtor, como menor custo de aplicação pela diminuição do tempo gasto no deslocamento para abastecimento, no tempo para o tratamento da área, na circulação do equipamento dentro da área, possibilidade de redução do número de equipamentos, e pela disponibilidade do trator para outras atividades na propriedade, além de reduzir a depreciação do conjunto trator pulverizador e consequentemente o custo de produção.

A produtividade média e o peso médio de bulbos comerciais (acima de $4 \mathrm{~cm}$ de diâmetro) não apresentaram diferenças estatisticamente significativas entre os diferentes tratamentos químicos em relação à testemunha (Tabela 3). Resultados semelhantes foram obtidos por Gonçalves (1996) que não observou aumento de produtividade para a cultura da cebola, com o uso de con- 
trole químico para tripes. Lorini \& Ferreto (1991) também não obtiveram diferenças de produtividade em cebola usando intervalos de aplicação de inseticida a 3, 7, 14 e 21 dias. Em trabalhos realizados no Brasil com nível de dano econômico de tripes em cebola tem-se observado que a planta pode tolerar níveis populacionais entre 15 a 25 tripes/ planta (Domiciano et al. 1993); 15 ninfas/planta antes de bulbificar e 30 após essa fase (Gonçalves, 1997a); e seis tripes/folha (Dória et al. 1998) sem prejuízos à produtividade. Portanto, apesar da presença da praga é possível obter produção de bulbos com padrão comercial e, nem sempre, o controle químico aumenta a produtividade da cultura. Esta questão tem sido discutida com extensionistas e agricultores da região do Alto Vale do Itajaí, SC, no sentido de reduzir o uso de inseticidas ou até mesmo suprimí-lo num contexto de produção orgânica da cultura.

\section{AGRADECIMENTOS}

Ao técnico Agrícola Marcelo Pitz e sua equipe pelo apoio e dedicação na condução do trabalho.

\section{LITERATURA CITADA}

ABBOTT, W.S. A method of computing the effectiveness of an insecticide. Journal of Economic Entomology. v. 18, p. 265 - 266, 1925.

CAVALCANTE, R.D.; ALMEIDA, P.R.; CAMPOS, T.B.; BITRAN, E.A. Controle de Thrips tabaci Lind. em cebola, com inseticidas clorados e fosforados. Horticultura Brasileira, Brasília, v. 4, n. 2, p. 46, 1986.
CARMAN, G.E. Spraying procedures for pest control on citrus. In: Ciba. Geigy. Agrochemicals. Basle, Tech. Monography, v. 4, p. $28-34,1975$

DOMICIANO, N.L.; OTA, A.Y.; TEDARDI, C.R. Momento adequado para controle químico de tripes, Thrips tabaci Lindeman, 1888 em cebola, Allium cepa L. Anais da Sociedade Entomológica do Brasil, v. 22, n. 1, p. 77-83, 1993.

DÓRIA, H.S.O.; GONÇALVES, K.C.; GONÇALVES, T.D.; FERNANDES, O.A.; BRAZ, L.T.; BARBOSA, J.C. Determinação do nível de dano econômico de Thrips tabaci (Thysanoptera, Thripidae) em cebola (Allium сера). In: CONGRESSO BRASILEIRO DE ENTOMOLOGIA, 17., 1998, Rio de Janeiro. Resumos ... Rio de Janeiro: UFRRJ/SEB, 1998. p. 337.

EMPASC/ACARESC. Sistema de produção para cebola . Florianópolis, 1991. 51 p. (Sistemas de Produção, 16).

GONÇALVES, P.A.S. Determinação de dano de Thrips tabaci Lind. em cultivares de cebola. Pesquisa Agropecuária Brasileira, Brasília, v. 31, n. 3, p. 173 - 179, 1996.

GONÇALVES, P.A.S . Determinação de nível de dano econômico de Thrips tabaci Lind. na cultura da cebola. In: CONGRESSO BRASILEIRO DE ENTOMOLOGIA, 16., 1997, Salvador. Resumos ... Salvador: EMBRAPACNPMF, 1997a. p. 287.

GONÇALVES, P.A.S. Flutuação populacional de tripes, Thrips tabaci Lind., em cebola em Ituporanga, Santa Catarina. Anais da Sociedade Entomológica do Brasil, Londrina, v. 26, n. 2, p. 365-369, $1997 \mathrm{~b}$.

GONÇALVES, P.A.S.; GUIMARÃES, D.R. Controle do tripes da cebola. Agropecuária Catarinense, Florianópolis, v. 8, n. 2, p. 44 46, 1995.

GUEDES, A.C.; PAGLARIN, R.C.; LINK, D. Avaliação de inseticidas para controle de tripes (Thrips tabaci Lindemann), na cultura da cebola (Allium cepa L.). Revista do Centro de Ciências Rurais, Santa Maria, v. 12, n. 4, p. 239 - 246, 1982.
HOWLAND, A.F.; WILCOX, J. Evaluation of new insecticides for control of thrips. Journal of Economic Entomology, v. 59, n. 4, p. 969 971, 1966.

LEWIS, T. Thrips: their biology, ecology, and economic importance. New York: Academic Press, 1973, 349 p.

LORCA, F.L. Experimentos de control del trips de la cebolla (Thrips tabaci Lind.) Agric. Tec. Chile, v. 18, n. 1, p. 17 - 23, 1958.

LORINI, I.; FERRETO, M. Avaliação de danos de Thrips tabaci Lindemann, 1888 (Thysanoptera: Thripidae) na cultura da cebola. Anais da Sociedade Entomológica do Brasil, v. 20, n. 2, p. 271-275, 1991.

MAYER, D.F.; LUNDEN, J.D.; RATHBONE, L. Evaluation of insecticides for Thrips tabaci (Thysanoptera: Thripidae) and effects of thrips on bulb onions. Journal of Economic Entomology, v. 80, p. 930 - 932, 1987.

MAYEUX, H.S.; WENE, G.P. Control of Onion Thrips with Low Volume Sprays. Journal of Economic Entomology, v. 43, n. 6, p. 908 912, 1950.

MOTE, U.N. Control of onion thrips (Thrips tabaci Lind.). Pesticides, v. 10, n. 7, p. $42-$ 43, 1976.

RAHEJA, A.K. Onion thrips and their control in Northern Nigeria. Samaru Agric. Newsletter, v. 15, n. 2 , p. $82-86,1973$.

ROSSITER, P.D.; GIESEMANN, K.J. Inseticides to control onion thrips (Thrips tabaci Lind.) Quensland Journal of Agricultural and Animal Sciences, v. 33, n. 1, p. 31 - 38, 1976.

SATO, M.E. Avaliação de dano e controle do Thrips tabaci Lindemann, 1888, na cultura da cebola (Allium cepa L.). Piracicaba: ESALQ 1989, 93 p. (Tese mestrado).

SAINI, R.K.; DAHIYA, A.S.; VERMA, A.N. Field evaluation of some insecticides against onion thrips, Thrips tabaci (Lindemann, 1888) (Thysanoptera: Thripidae). Haryana Agriculture University Journal Research, v. 19, n. 4, p. 236 - 342, 1989.

SHARMA, V.P.; SRIVASTAVA, B.P. Dusts and sprays keep onion thrips away. Indian Farming, v. 15, n. 9, p. 24 - 25, 1965. 